\title{
Synthesis of a Novel Biodegradable Polyurethane with Phosphatidylcholines
}

\author{
Jun Cao ${ }^{1}$, Niancao Chen ${ }^{1}$, Yuanwei Chen ${ }^{1}$ and Xianglin Luo ${ }^{1,2, *}$ \\ 1 College of Polymer Science \& Engineering, Sichuan University, Chengdu 610065, China; \\ E-Mails: caojun198610301981@126.com (J.C); chenncscu@gmail.com (N.C.); \\ chenyuanweizm@163.com (Y.C.) \\ 2 State Key Lab of Polymer Materials and Engineering, Sichuan University, Chengdu 610065, China \\ * Author to whom correspondence should be addressed; E-Mail: luoxl_scu@126.com; \\ Tel.: +86-28-854-661-66; Fax: +86-28-854-054-02.
}

Received: 17 March 2010; in revised form: 31 March 2010 / Accepted: 1 April 2010 /

Published: 26 April 2010

\begin{abstract}
A novel polyurethane was successfully synthesized by chain-extension of biodegradable poly (L-lactide) functionalized phosphatidylcholine (PC) with hexamethylene diisocyanate (HDI) as chain extender (PUR-PC). The molecular weights, glass transition temperature $(\mathrm{Tg})$ increased significantly after the chain-extension. The hydrophilicity of PUR-PC was better than the one without PC, according to a water absorption test. Moreover, the number of adhesive platelets and anamorphic platelets on PUR-PC film were both less than those on PUR film. These preliminary results suggest that this novel polyurethane might be a better scaffold than traditional biodegradable polyurethanes for tissue engineering due to its better blood compatibility. Besides, this study also provides a new method to prepare PC-modified biodegradable polyurethanes.
\end{abstract}

Keywords: polyurethanes; phosphatidylcholine; biodegradable; blood compatibility; polymer; surface

\section{Introduction}

Biomaterials for tissue regeneration need to be biocompatible as well as biodegradable in vivo. Polyurethanes (PURs) are widely used in biomedical fields due to their excellent mechanical properties [1]. In order to further improve their blood compatibility, lots of modification methods have 
been developed. Among them, the imitation of cell membrane by introducing PC into PURs is a very effective way [2-5]. For example, aryl azides consisting of a photoactivatable 4-azidobenzoyl group and a PC end group have been synthesized and then the PC-containing aryl azides were coupled to poly (etherurethane) surfaces. Many studies purport to assess blood compatibility by counting adherent platelets and changing the morphology of the platelets on the surfaces of materials. What is more, as soon as the blood contacts a material, the protein starts to adsorb onto the materials surface and change their conformation. Thus, the platelets interact with these and eventually starting adhering and spreading onto the protein layer. The platelets in the state are activated to release blood coagulation factors [6]. So, the blood compatibility of the modified PURs was evaluated with a thrombin generation assay and a platelet adhesion study. The maximum thrombin concentration was lower than that of the original PURs. Meanwhile, the clotting time of the blood was also extended and platelet adhesion was effectively reduced on the modified PURs [4,5]. However, all the membrane-like PURs reported so far are seldom likely to degrade, which becomes the main limitation for their further application in tissue engineering. Although biodegradable PURs without PC have been intensively investigated in recent years, such as poly (lactide) (PLA), poly(glycolic acid) (PGA), poly(caprolactone) (PCL) and their copolymers etc. [7-11], they are not so blood compatible compared to PC-containing PURs.

In this study, PUR-PC, a novel polyurethane with PC groups in the biodegradable soft segments (PC-S) was synthesized. Meanwhile, polyurethane (PUR), as a contrast, was synthesized in the same way using PLLA as soft segment (S). The molecular structure and molecular weight were analyzed by proton NMR ( $\left.{ }^{1} \mathrm{H}-\mathrm{NMR}\right)$, Fourier transform infrared spectroscopy (FT-IR) and Size exclusion chromatograph (SEC), respectively. Differential scanning calorimetry (DSC) was used to monitor the thermal properties. The hydrophilicity and in vitro blood compatibility were evaluated by water absorption test and platelet adhesion test respectively.

\section{Experiment}

The synthesis of PC-S was carried out under vacuum at $120{ }^{\circ} \mathrm{C}$ for $36 \mathrm{~h}$ by using glycerophosphatidylcholine as initiator (Figure1) [12]. Then PC-S was dried for two hours under vacuum before introducing it into a three-neck flask with toluene, a magnetic stir bar and stannous octoate (catalyst). The prescribed amount of hexamethylene diisocyanate (HDI) in toluene was added into the flask dropwise (molar ratio of $\mathrm{HDI} /$ prepolymer $=3$ ). The reaction progressed under a $\mathrm{N}_{2}$ atmosphere at $120{ }^{\circ} \mathrm{C}$ for $7 \mathrm{~h}$. The products were precipitated in ethanol and dried under vacuum to a constant weight. Synthesis of PUR without PC was carried out in a similar way. Figure 1 illustrates the synthesis of PC-S and PUR-PC.

Polymers were characterized by ${ }^{1} \mathrm{H}-\mathrm{NMR}$ (Bruker AV II-400 MHz), FT-IR (Nicolet 560). SEC was performed on equipment composed of a Waters 510 HPLC pump, a Waters 410 differential refractometer and a PLgel $5 \mathrm{~mm}$ mixed-C $60 \mathrm{~cm}$ column, the mobile phase being tetrahydrofuran (THF) and the flow rate $1 \mathrm{~mL} / \mathrm{min}$. The number-average $(\mathrm{Mn})$ and weight-average $(\mathrm{Mw})$ molecular weights were expressed with respect to polystyrene standards obtained from Polysciences. DSC (Netzsch DSC204) was used to characterize the glass transition temperature (Tg). 
Figure 1. Synthetic route to PUR-PC.
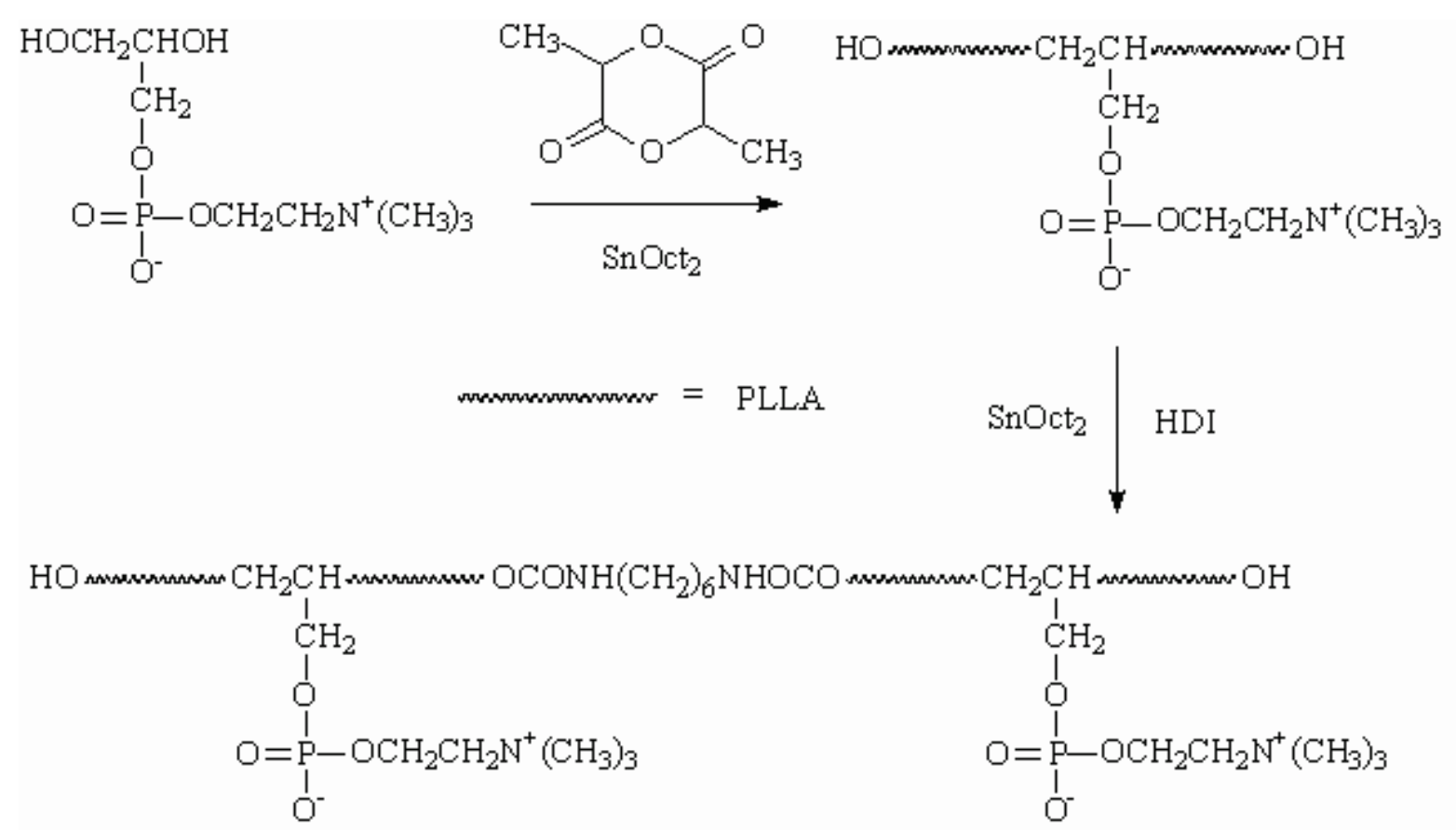

(PUR-PC)

Water absorption was used to evaluate the hydrophilicity. It is defined as the weight percentage of water in wet polymer films. The films were prepared by casting $1 \mathrm{wt} \%$ polymer solution (in chloroform) onto polyethyleneglycol terephthalate (PET) substrates $(1 \mathrm{~cm} \times 1 \mathrm{~cm})$. After vacuum drying for 2 days, the films were weighed $\left(W_{0}\right)$ and placed in distilled water. They were recovered at different time intervals, carefully wiped with filter paper and weighed $\left(W_{1}\right)$ again. The water absorption was calculated with the following equation:

$$
\text { Water uptake }(\%)=\left(W_{1}-W_{0}\right) / W_{0} \times 100 \%
$$

The blood platelet adhesion evaluation was carried out according to the method reported by reference [2]. Films were sputter-coated with gold in vacuum and observed by using a scanning electron microscope (SEM, JSM- 5900 LV, Jeol Ltd., Japan).

\section{Experimental Results and Discussion}

\subsection{The Structure and Characterization of the POLYMERs}

To obtain PC-modified polyurethane, both surface modification and bulk modification methods are developed by researchers. In the bulk modification, PC is normally introduced by a graft reaction after chain extension. In addition, PC could also be introduced during the chain-extending reaction. In this situation, PC-containing chemicals served as chain-extenders [3]. In this study, a biodegradable polyurethane with PC (PUR-PC) was synthesized according to the synthetic route demonstrated in Figure 1. The synthesis strategy is similar to that of the normal polyurethane, which includes the synthesis of soft segments initially and then the chain-extension. PLLA with PC (PLLA/PC) took the role of the soft segment, the part of polylactide (PLA) is widely recognized to be biodegradable in the 
physiological environment. PLLA/PC was prepared by ring-opening polymerization of lactide with glycerophosphorylcholine as initiator (see Figure 1). The molecular structures were confirmed by the results of FT-IR, ${ }^{13} \mathrm{C}$-NMR and ${ }^{1} \mathrm{H}-\mathrm{NMR}$ analysis which were consistent with those reported before [12] (data not shown). Chain extension was then carried out after the confirmation. HDI served as the chain-extender (see Figure1). FT-IR and ${ }^{1} \mathrm{H}-\mathrm{NMR}$ were also employed to analyze the molecular structure of the obtained polyurethane. Figure 2 shows the ${ }^{1} \mathrm{H}-\mathrm{NMR}$ spectrum of PUR-PC. Signals at 1.55 and 5.2 were ascribed to the $-\mathrm{CH}_{3}$ and $-\mathrm{CH}_{2}$ - groups in the PLLA blocks in the soft segments, respectively. The typical peaks of $\mathrm{PC}$, including $-\mathrm{N}^{+}\left(\mathrm{CH}_{3}\right)_{3},-\mathrm{OPOCH}_{2}$ and $-\mathrm{N}^{+} \mathrm{CH}_{2}$ - were found at $3.25,3.43$ and 4.3 , respectively [12,13]. Signals at 1.3 and 3.15 were assigned to the $-\mathrm{CH}_{2}$ - units of HDI in the hard segments [14]. In FT-IR spectra, the characteristic absorption peak of $-\mathrm{N}^{+}\left(\mathrm{CH}_{3}\right)_{3}$ in the soft segment was observed at $970 \mathrm{~cm}^{-1}[12,13]$. The peaks at $1,528 \mathrm{~cm}^{-1}$ and $3,405 \mathrm{~cm}^{-1}$ were attributed to $-\mathrm{NH}-$ and NH-CO, respectively, which were absent in the spectrum of soft segments.

Figure 2. ${ }^{1} \mathrm{H}-\mathrm{NMR}$ spectrum of PUR-PC.

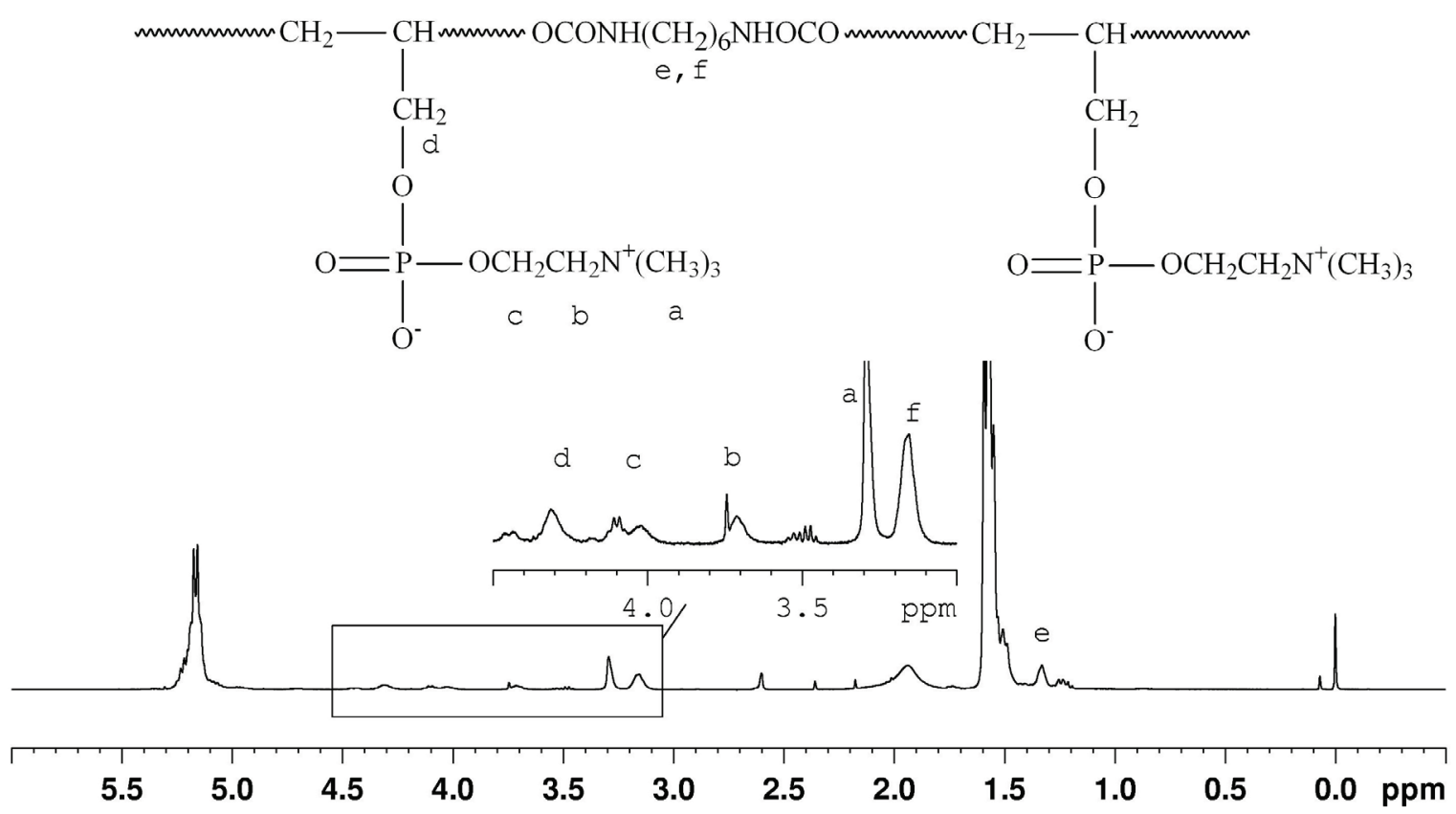

The molecular weights $\left(\mathrm{M}_{\mathrm{W}}\right)$ of the synthesized polymers were examined by SEC. As shown in Table 1, the number average M (Mn) of the soft segment PC-S was about 2,500, it increased to more than 5,000 after the chain-extending reaction. This result once again confirmed the successful synthesis of PUR-PC polymer. DSC was employed to test the thermal properties of the polymers. The glass transition temperature $(\mathrm{Tg})$ values of the soft segment of the PUR-PC was only about $28{ }^{\circ} \mathrm{C}$. It increased to $53.3{ }^{\circ} \mathrm{C}$ after the chain extension (Table 1). These might be explained by the improved molecular weight and by the strong interaction between molecular chains resulting from the polarity of the $\mathrm{O}=\mathrm{C}=\mathrm{N}$ groups in the polyurethane. 
Table 1. Characterization of the polymers.

\begin{tabular}{|c|c|c|c|c|}
\hline Polymers & $M^{a)}$ & $\mathbf{M w}^{\mathbf{b})}$ & PDI ${ }^{\text {b) }}$ & $\operatorname{Tg}\left({ }^{\circ} \mathrm{C}\right)$ \\
\hline PC-S ${ }^{c)}$ & 2490 & 3680 & 1.48 & 27.7 \\
\hline PUR-PC & 5460 & 8270 & 1.52 & 53.3 \\
\hline PC-S' & 3153 & 3996 & 1.267 & 38.6 \\
\hline PUR-PC' & 8234 & 14532 & 1.765 & 55.2 \\
\hline PUR & 24380 & 57680 & 2.37 & 44.7 \\
\hline \multicolumn{5}{|c|}{ a) Calculated by SEC results; } \\
\hline \multicolumn{5}{|c|}{ W/IVII, } \\
\hline c) The & of $\mathrm{P}$ & $/ \mathrm{PC} \mathrm{m}$ & ed $\mathrm{w}$ & \\
\hline
\end{tabular}

\subsection{Water Absorption and Blood Platelet Adhesion Test}

Water absorption was used to evaluate the hydrophilicity of the polymers. As shown in Figure 3, the water absorption value of PUR-PC was about $6 \% 2$ days after being in water. It's evidently higher than that of the PUR without PC. Moreover, this difference increased over time durin the period of 8 days. At the end of the test period, the water absorption value of PUR-PC was about five times of the PUR water absorption value. This suggested that biodegradable PUR-PC was more hydrophilic than PUR. Normally, the introduction of PC would improve the hydrophilicity of polymers. This was ascribed to strong affinity between PC and water molecules. Various published papers have proved that the PC groups migrate to the surfaces of polymers when contacted with water and consequently combined with water molecules $[12,13,15,16]$.

Figure 3. Water absorption of the synthesized copolymers.

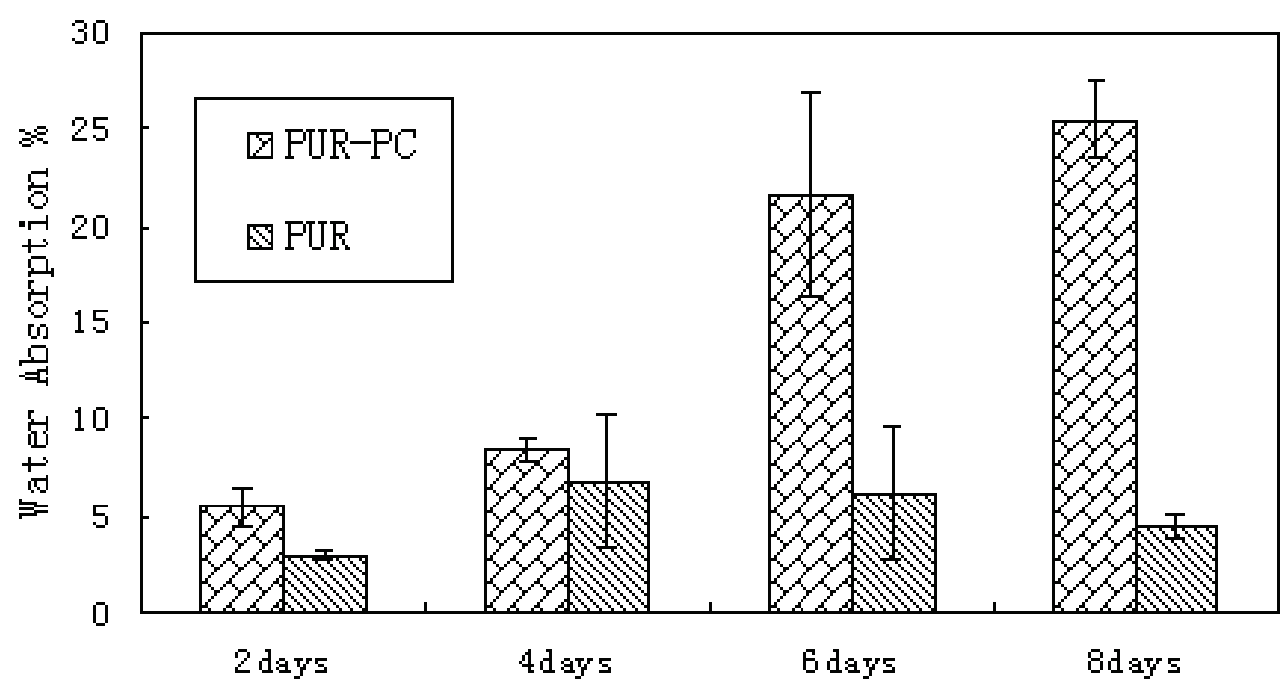


Figure 4 shows the adherent platelets (white dots) on the polymer surface after contacting with rabbit platelet rich plasma (PRP) for $60 \mathrm{~min}$. A large number of platelets were observed to have adhered on PUR. In contrast; much less platelets could be seen on PUR-PC surface. Moreover, cell morphologies were found to be different on both of the polyurethanes films. Some platelets on PUR protruded flake pseudopodias, which made them larger than the static platelets (black arrows in Figure 4). In contrast, almost all the platelets on PUR-PC remained in their inactive round shape. Therefore, it could be deduced that PUR-PC could diminish adhesion and activation of platelets on PUR surface. This might be attributed to the affinity between the water molecules and the polar PC in PUR-PC. And this led to the formation of a layer of water on the surfaces of polymers in aqueous environment. The water layer suppressed the non-specific protein adsorption and subsequent platelet adhesion, as reported in references [2,4,5].

Figure 4. SEM pictures of platelets adhered on polymers surface. Black arrows: Anamorphic platelets: $\mathrm{a}, \mathrm{b}-\mathrm{PUR}-\mathrm{PC} ; \mathrm{c}, \mathrm{d}-\mathrm{PUR}$.
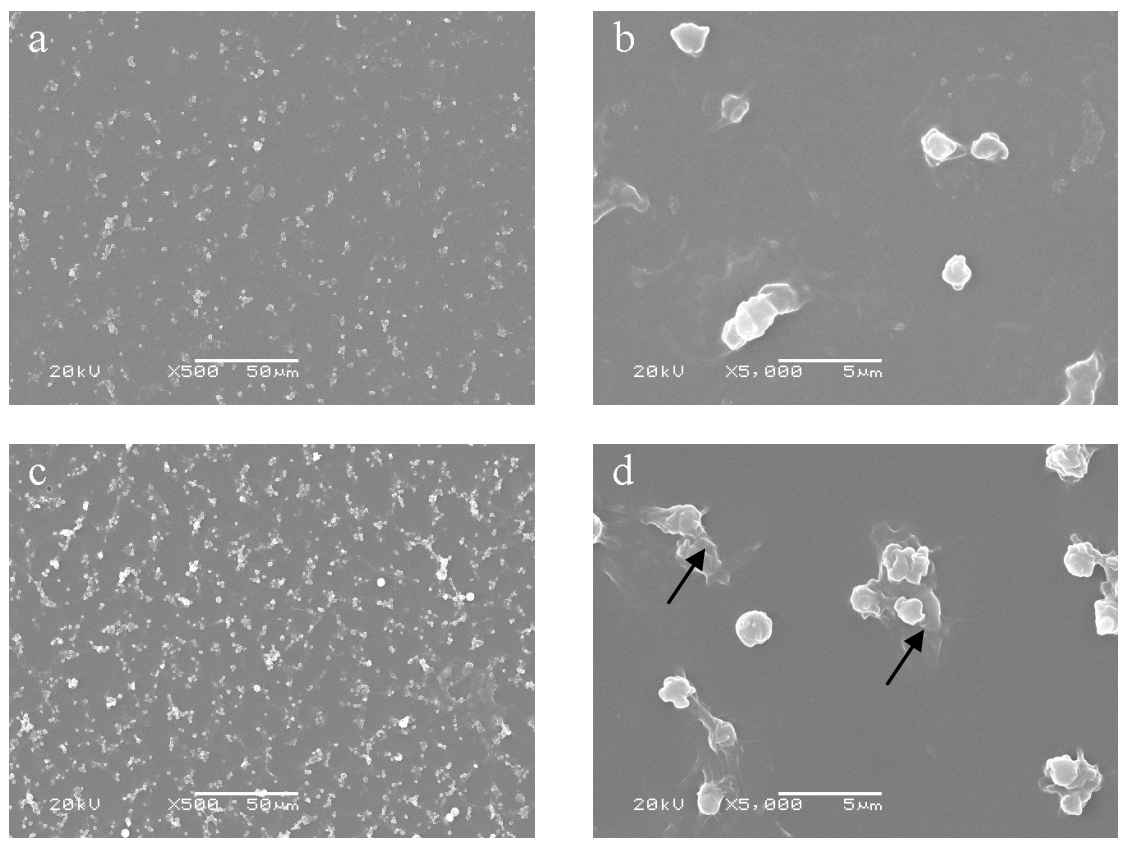

\section{Conclusions}

A novel polyurethane with PC in its biodegradable soft chains was successfully synthesized. The molecular weight and glass transition temperature $(\mathrm{Tg})$ increased significantly after the chain-extension. The hydrophilicity of PUR-PC was better than that of PUR. Moreover, the number of adhesive platelets and anamorphic platelets on PUR-PC were both less than those on PUR. These preliminary results suggested that the novel polyurethane might be a better scaffold than traditional biodegradable PUR for tissue engineering due to its better blood compatibility. This study also provides a new method to prepare PC-modified biodegradable PUR. 


\section{Acknowledgements}

This study was financially supported by NSFC (No. 50473025), the International S\&T Cooperation Program of China (No. 2006DFA53470) and the France-China advanced research program (No. PRA MX-05-06).

\section{References and Notes}

1. Guelcher, S.A. Biodegradable polyurethanes: synthesis and applications in regenerative medicine. Tissue Eng.: B 2008, 14, 3-17.

2. Korematsua, A.; Takemotob, Y.; Nakaya, T.; Inouea, H. Synthesis, characterization and platelet adhesion of segmented polyurethanes grafted phospholipid analogous vinyl monomer on surface. Biomaterials 2001, 23, 263-271.

3. Baumgartner, J.N.; Yang, C.Z.; Cooper, S.L. Physical property analysis and bacterial adhesion on a series of phosphonated polyurethanes. Biomaterials 1997, 18, 831-837.

4. van der Heiden, A.P.; Goeggels, D.; Pijpers, A.P.; Koole, L.H. A photochemical method for the surface modification of poly (etherurethanes) with phosphorylcholine-containing compounds to improve hemocompatibility. J. Biomed. Mater. Res. 1997, 37, 282-290.

5. van der Heiden, A.P.; Willems, G.M.; Lindhout, T.; Pijpers, A.P.; Koole, L.H. Adsorption of proteins onto poly(ether urethane) with a phosphorylcholine moiety and influence of preadsorbed phospholipid. J. Biomed. Mater. Res. 1998, 40, 195-203.

6. Ratner, B.D. The catastrophe revisted: Blood compatibility in the 21 st Century. Biomaterials 2007, 28, 5144-5147.

7. Guan, J.J.; Fujimoto, K.L.; Sacks, M.S.; Wagner, W.R. Preparation and characterization of highly porous, biodegradable Polyurethane scaffolds for soft tissue applications. Biomaterials 2005, 26, 3961-3971.

8. Alperina, C.; Zandstrab, P.W.; Woodhouse, K.A. Polyurethane films seeded with embryonic stem cell-derived cardio myocytes for useincardiac tissue engineering applications. Biomaterials 2005, 26, 7377-7386.

9. Gorna, K.; Gogolewski, S. Biodegradable porous polyurethane scaffolds for tissue repair and regeneration. J. Biomed. Mater. Res. 2006, 79A, 128-138.

10. Wang, W.S.; Ping, P.; Chen, X.S.; Jing, X.B. Biodegradable polyurethane based on random copolymer of L-lactide and $\varepsilon$-caprolactone and its shape-memory property. J. Appl. Polym. Sci. 2007, 104, 4182-4187.

11. Da Silva, G.R.; Da Silva, Cunha, A.S., Jr.; Ayres, E.; Orefice, R.L. Effect of the macromolecular architecture of biodegradable polyurethanes on the controlled delivery of ocular drugs. J. Mater. Sci.: Mater. Med. 2009, 20, 481-487.

12. Iwasaki, Y;; Tojo, Y.; Kurosaki ,T.; Nakabayashi, N. Reduced adhesion of blood cells to biodegradable polymers by introducing phosphorylcholine moieties. J. Biomed. Mater. Res. 2003, 65A, 164-169.

13. Chen, N.C.; Chen, Y.W.; Wang, L.J.; Luo, X.L. Preparation, characterization and blood compatibility of polylactide-based phospholipid polymer. J. Mater. Sci. 2009, 44, 6317-6320. 
14. Woo, S.I.; Kim, B.O.; Jun, H.S.; Chang, H.N. Polymerization of aqueous lactic acid to prepare high molecular weight poly (lactic acid) by chain-extending with hexamethylene diisocyanate. Polym. Bull. 1995, 35, 415-421.

15. Nederberg, F.; Bowden, T.; Hilborn, J. Induced surface migration of biodegradable phosphorylcholine functional poly (trimethylene carbonate). Polym. Adv. Technol. 2009, 16, 108-112.

16. Yang, S.; Zhang, S.P.; Winnik, F.M.; Mwale, F.; Gong, Y.K. Group reorientation and migration of amphiphilic polymer bearing phosphorylcholine functionalities on surface of cellular membrane mimicking coating. J. Biomed. Mater. Res. 2009, 84A, 837-841.

(C) 2010 by the authors; licensee MDPI, Basel, Switzerland. This article is an open-access article distributed under the terms and conditions of the Creative Commons Attribution license (http://creativecommons.org/licenses/by/3.0/). 